\title{
A randomised controlled trial of a lifestyle behavioural intervention for patients with low back pain, who are overweight or obese: study protocol
}

Amanda Williams ${ }^{1,2,3^{*}}$, John Wiggers ${ }^{1,2,3}$, Kate M. O'Brien ${ }^{1,2,3}$, Luke Wolfenden ${ }^{1,2,3}$, Serene Yoong ${ }^{1,2,3}$, Elizabeth Campbell ${ }^{1,2,3}$, Emma Robson ${ }^{1}$, James McAuley ${ }^{4,5}$, Robin Haskins ${ }^{6}$, Steven J. Kamper ${ }^{7}$

and Christopher M. Williams ${ }^{1,2,3,7}$

\begin{abstract}
Background: Low back pain is a highly prevalent condition with a significant global burden. Management of lifestyle factors such as overweight and obesity may improve low back pain patient outcomes. Currently there are no randomised controlled trials that have been conducted to assess the effectiveness of lifestyle behavioural interventions in managing low back pain. The aim of this trial is to determine if a telephone-based lifestyle behavioural intervention is effective in reducing pain intensity in overweight or obese patients with low back pain, compared to usual care.

Methods/Design: A randomised controlled trial will be conducted with patients waiting for an outpatient consultation with an orthopaedic surgeon at a public tertiary referral hospital within New South Wales, Australia for chronic low back pain. Patients will be randomly allocated in a 1:1 ratio to receive a lifestyle behavioural intervention (intervention group) or continue with usual care (control group). After baseline data collection, patients in the intervention group will receive a clinical consultation followed by a 6-month telephone-based lifestyle behavioural intervention (10 individually tailored sessions over a 6-month period) and patients in the control group will continue with usual care. Participants will be followed for 26 weeks and asked to undertake three self-reported questionnaires at baseline (pre-randomisation), week 6 and 26 post randomisation to collect primary and secondary outcome data. The study requires a sample of 80 participants per group to detect a 1.5 point difference in pain intensity (primary outcome) 26 weeks post randomisation. The primary outcome, pain intensity, will be measured using a 0-10 numerical rating scale.
\end{abstract}

Discussion: The study will provide robust evidence regarding the effectiveness of a lifestyle behavioural intervention in reducing pain intensity in overweight or obese patients with low back pain and inform management of these patients.

Trial registration number: Australian New Zealand Clinical Trials Registry, ACTRN12615000478516, Registered 14/05/2015.

Keywords: Low back pain, Obesity, Lifestyle, Telephone, Randomised controlled trial, Protocol

\footnotetext{
* Correspondence: Amanda.J.Williams@hnehealth.nsw.gov.au

'Hunter New England Population Health, Locked Bag 10, Wallsend NSW,

2287, Australia

${ }^{2}$ Hunter Medical Research Institute, Locked Bag 1, Hunter Region Mc, NSW

2310 Newcastle, Australia

Full list of author information is available at the end of the article
} 


\section{Background}

Low back pain is a common condition and poses significant burden to individuals and society. Globally, the median point prevalence of low back pain has been reported to be $15 \%$ [1] and the global lifetime prevalence as high as $84 \%$ [2]. The latest Global Burden of Disease Study (2013) reported over 651 million cases of low back pain in 2013, which is the leading cause of disability measured [3]. As a consequence, low back pain represents a considerable economic burden. Direct costs of care are reported to be more than \$AU4.7 billion in Australia (2012 values), more than $£ 1.6$ billion in the United Kingdom (1998 values) and as much as \$US90 billion in the United States (1998 values) $[4,5]$.

While the aetiology of low back pain remains unclear, it is now widely accepted that effective treatment for low back pain requires consideration of the psychological and behavioural factors. Several lifestyle behavioural factors are reported to be associated with an increased prevalence and persistence of low back pain including weight, sleep disturbance, psychological distress, and beliefs. Among the most compelling evidence is the association between overweight and obesity and low back pain $[6,7]$. One meta-analysis which included 33 cross-sectional and cohort studies, found significant associations between overweight or obesity and a range of low back pain outcomes. Data from the cohort studies showed that overweight or obesity is associated with an increased 12-month prevalence of low back pain $(n=6828$; OR 1.21, $95 \%$ CI: 1.07 , 1.37), increased risk of chronic low back pain (defined as longer than 3 months in duration; OR 1.43, 95 \% CI: 1.28, 1.60), and higher rates of health care seeking for low back pain (OR 1.56, 95 \% CI: 1.46, 1.67) [7]. Similar associations have been reported for body mass index (BMI) [8]. While the association between physical activity and diet and low back pain is less consistent, these are key drivers of weight gain [9]. Certainly, patients with low back pain who are overweight or obese, are likely to have more complex health needs requiring focus on a holistic lifestyle and behavioural approach to management.

Given these widely reported associations between lifestyle behavioural factors and low back pain, it is suggested that targeting these as part of low back pain management could improve patient outcomes $[7,10,11]$. While international guidelines for weight management recommend behavioural modification interventions as the preferred approach to managing weight loss and healthy lifestyle there is limited evidence to guide such care in patients with low back pain [9]. Several systematic reviews have found no randomised controlled trials (RCT) reporting the effectiveness of lifestyle behavioural interventions in managing persistent low back pain $[10,12]$. To the author's knowledge only one prepost study of a 52 week medically supervised weight loss program for obese patients with low back pain has been conducted. The study found a statistically significant weight loss of $15.3 \mathrm{~kg}(95 \%$ CI: 7.8, 22.8) was associated with a significant improvement in pain related disability (Oswestry Disability Index (ODI) baseline $31.9 \pm 17.7$, follow-up 27.1 $\pm 20.9, p=0.009)$ [13]. While promising, there is a need to test the effectiveness of lifestyle behavioural interventions on low back pain outcomes in robust RCTs.

Given the large numbers of patients who suffer from low back pain and are overweight or obese [6, 7], an important consideration is to provide cost effective interventions that are accessible to a large proportion of overweight patients at relatively lower cost to patients. Telephone-based interventions as a treatment delivery modality has potential to provide greater access to treatment for patients, and overcomes barriers to accessing continued care, including time and travel requirements to attend face-to-face appointments, and flexible scheduling of contact [14]. Importantly, telephone-based interventions that include behavioural modification and adjunct psychological strategies are consistently shown to be as effective as face-to-face interventions in achieving weight loss $[15,16]$. For the key determinants of weight loss; physical activity and diet modification, telephone-based interventions have also been shown to be more cost-effective compared to clinical faceto-face practices [14].

The primary objective of the study is to determine if a telephone-based lifestyle behavioural intervention is effective in reducing pain intensity in overweight or obese patients with low back pain, compared to usual care. Secondary objectives are to investigate if the intervention improves key secondary outcomes: disability and function, anthropometry (weight, BMI, waist circumference), quality of life, diet, physical activity and health care utilisation, compared to usual care.

\section{Methods \\ Study design and setting}

The study will employ a parallel group randomised controlled design (Fig. 1), as part of a cohort multiple RCT [17]. This pragmatic design utilises participants from our existing cohort of routine service; patients are randomised to be offered a new clinical intervention (intervention group) or to remain part of the cohort (control group). The control group is not aware of the intervention trial and thus act as a real world usual care comparison. This protocol adheres to the requirements of the Standard Protocol Items: Recommendations for Intervention Trials (SPIRIT) guidelines and is prospectively registered with the Australian New Zealand Clinical Trials Registry (ACTRN12615000478516). The trial will be undertaken in the Hunter New England Local Health District, New South Wales (NSW), Australia. Ethical approval has been obtained from the Hunter New England Human Research Ethics Committee 


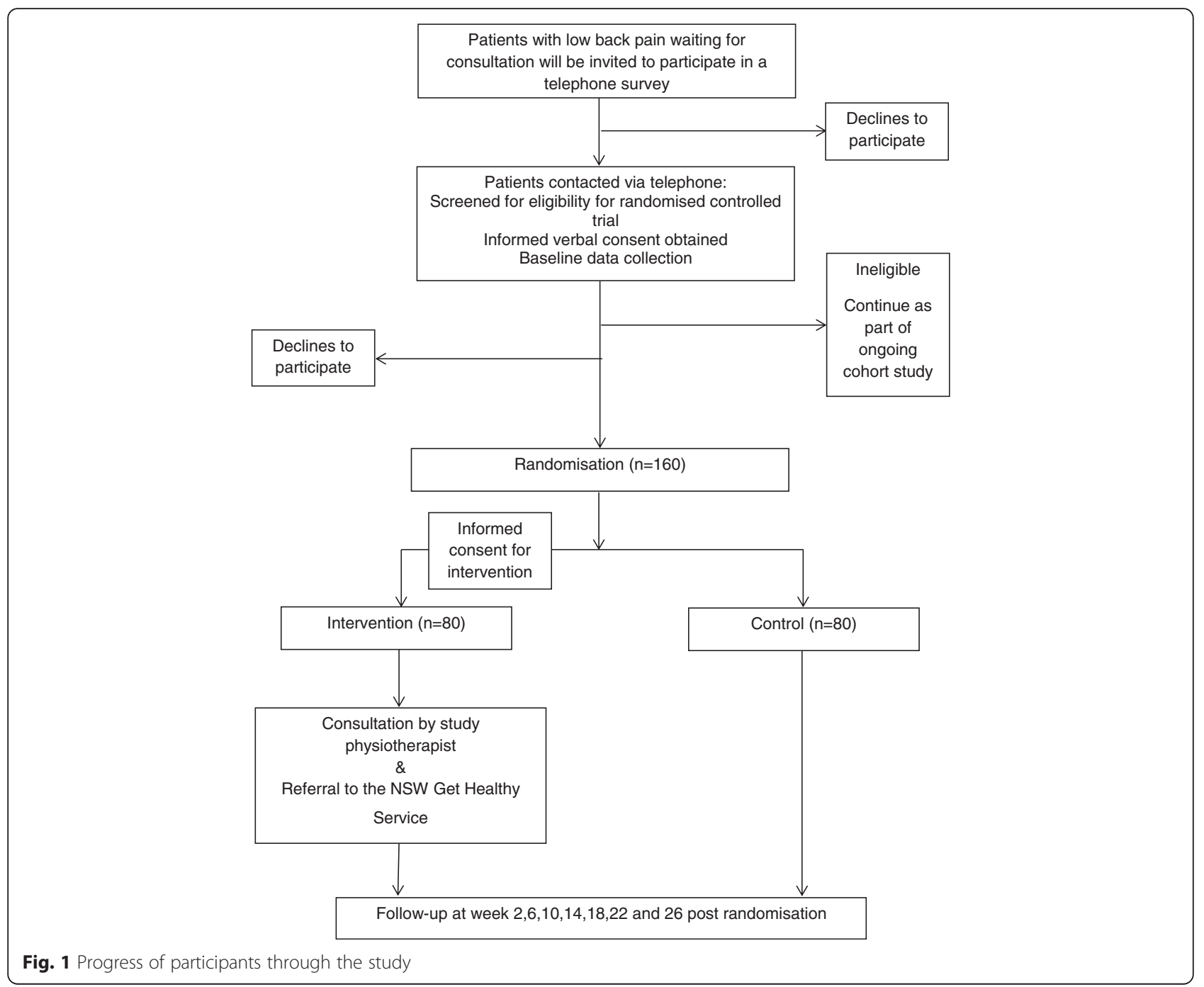

(approval No. 13/12/11/5.18) and the University of Newcastle Human Research Ethics Committee (approval No. H-2015-0043).

\section{Population and recruitment}

One hundred and sixty patients waiting for an outpatient orthopaedic consultation at a public tertiary referral hospital within NSW for non-specific low back pain will be recruited. All patients over 18 years of age waiting for an outpatient consultation for low back pain will be sent an information letter to invite participation in a telephone survey as part of the ongoing cohort study. Patients will be asked to contact the researchers if they do not wish to participate or can refuse upon receipt of the telephone call. Patients consenting to the telephone survey will then be screened for eligibility for the RCT by a trained interviewer, invited to participate if eligible for the study and asked to complete the baseline survey at the time of the call.

To be eligible participants must meet the following criteria:

- Chronic low back pain defined as: pain in the lower back (i.e. between the 12th rib and buttock crease) with/without leg pain and a duration of longer than 3 months since the onset of pain [18];

- Aged 18 years or older;

- Classified as overweight or obese with a BMI of $\geq 27 \mathrm{~kg} / \mathrm{m}^{2}$ and $<40 \mathrm{~kg} / \mathrm{m}^{2}$ - based on self-reported weight and height;

- Have access to and can use a telephone;

- Low back pain severe enough to cause at least average low back pain intensity $\geq 3$ of 10 on a $0-10$ numerical rating scale (NRS) in the last week or 
moderate level of interference in activities of daily living (adaptation of item 8 on SF36).

Patients will be excluded if they meet the following criteria:

- Known or suspected serious pathology as the underlying cause of back pain (e.g. fracture, cancer, infection, inflammatory arthritis, cauda equine syndrome);

- A previous history of obesity surgery;

- Currently participating in any prescribed, medically supervised or commercial weight loss program;

- Back surgery in the last 6 months or booked in for surgery in the next 6 months;

- Unable to comply with the study protocol that requires them to, adapt meals or exercise, due to non-independent living arrangements;

- Any medical or physical impairment, apart from back pain, precluding safe participation in exercise such as uncontrolled hypertension, or morbid obesity (BMI $\geq 40$ );

- Cannot speak and read English sufficiently to complete the study procedures.

\section{Randomisation and blinding}

A randomisation schedule will be created a priori by an independent investigator using SAS 9.3 through the SURVEYSELECT procedure. Consenting patients who are eligible for the trial will be allocated, in a 1:1 allocation ratio, to either receive the lifestyle behavioural intervention at that time (intervention group) or remain as part of the cohort and be told they will be offered clinical services in 6 months (control group). To randomise patients, a trained interviewer will open a sealed opaque envelope containing group allocation. A staff member not involved in the study will prepare the envelopes. Patient progress through the study is outlined in Fig. 1.

All outcome assessors will be blind to group allocation.

\section{Treatments}

\section{Intervention group}

Patients randomised to the intervention group will be provided brief advice and education about the benefits of weight loss and physical activity for their conditions by trained telephone interviewers. Participants will then be invited to attend a one hour consultation with the study physiotherapist at Hunter New England Population Health, NSW, Australia and referred to the NSW Get Healthy Information and Coaching Service (GHS) [19, 20].

\section{Consultation}

The consultation will involve a low back pain clinical assessment and detailed low back pain education based on principles recommended by clinical practice guidelines. The consultation will also apply behaviour change techniques to support a healthy lifestyle and weight management for low back pain. This intervention content was informed by Self Determination Theory (SDT) [21, 22]. According to SDT autonomous behaviour rather than behaviour controlled by rewards, punishments or selfimposed pressures is more likely to result in long lasting behaviour change [22]. The constructs deemed integral in SDT to develop autonomous motivation include increasing 1) perceived competence (increase interest, enjoyment and importance) and 2) self-regulation (increase ability to direct behaviour to act in your long term best interest and in line with your values) [22]. The specific techniques used in the consultation to address these key constructs include: i) provision of education and reassurance to correct inappropriate pain beliefs and improve self-efficacy for self-management (i.e. provide information about the about the nature of the condition, that persistent low back pain is multifactorial with multiple influences and not usually the result of pathological damage), ii) acknowledging the consequences of unhealthy lifestyle factors (overweight, inactivity, poor diet, alcohol misuse, smoking, poor sleep) on low back pain, iii) provide general encouragement and examples of how improving lifestyle factors can influence pain outcomes and quality of life, iv) prompt commitment from the participant, v) acknowledge that monitoring of behaviours will be conducted throughout the program, vi) setting graded tasks to adopt better physical functioning and healthy behaviour (e.g. begin walking $30 \mathrm{~min}$ daily), vii) encourage self-monitoring of goals, viii) present the NSW GHS as a way to support ongoing behaviour change to improve low back pain and general health, ix) acknowledge general barriers that may reduce motivation to change lifestyle and adherence to the program (e.g. acknowledge fluctuating nature of condition and that high levels of pain are the result of a complex interaction of factors not just the result of increased activity, and discourage use of pain as a guide for progression of activity).

\section{Lifestyle behavioural intervention - The NSW Get Healthy Service}

Following the consultation, patients randomised to the intervention group will be referred to the established GHS [19]. The referral to the GHS will be provided to the service on the participants' behalf. The GHS is a free telephone-based government funded service to support individuals to modify their eating behaviours, increase their physical activity, reduce alcohol consumption and maintain a healthy weight or reduce their weight. The service was developed in response to evidence supporting the efficacy of telephone-based behaviour modification interventions and facilitates the translation of this evidence into a population wide approach [19]. A pre- 
post study assessing the effectiveness of the GHS in the general population reported significant reductions in weight, BMI, and waist circumference, and significant improvements in physical activity and nutrition-related behaviours [19].

The GHS service involves 10 individually tailored coaching calls delivered over a 6 month period by a qualified health professional including dieticians, exercise physiologists and psychologists [19]. The support provided is based on national guidelines including the Australian Guide to Healthy Eating and National Physical Activity Guidelines [19, 23], utilises motivational interviewing principles [19, 24], addresses health-related psychological blocks with Socratic questioning [25], and applies selfregulation principles including goal setting, overcoming barriers and creating sustainable changes [19]. The program is individually tailored to each patient with content targeted to address individual patient goals throughout the 6 months and phone calls scheduled according to the patient's preferences. These aspects are determined by the patient and health coach together, however calls are generally provided on a tapered schedule, with a higher intensity of calls $(n=6)$ made within the first three months of the program [19]. This schedule facilitates initiation of behaviour change in the first three months and maintenance and prevention of relapse in the second half of the program. In addition to the health coaching calls, participants receive an information booklet that provides additional information to support them during the program to achieve their goals, a coaching journal to record goals and actions, and access to online services to help track their progress. Medical clearance from a general practitioner will be obtained when required, as per existing service protocols [19].

All health coaches, regardless of multidisciplinary background, receive training to ensure they meet the requirements of the service and to promote consistency across the program. The service conducts audits of coaching quality as part of its quality improvement practices. To ensure the GHS health coaching is relevant for low back pain participants, health coaches will be provided additional training by a study investigator $(\mathrm{CW})$ in evidence-based management for low back pain $(2 \mathrm{~h}$ interactive training session) and provided with information resources to guide specific advice to be provided to study participants. The training session includes the topics of diagnosis, prognosis and evidence-based management strategies including the role of a healthy lifestyle and weight loss. The information provided is contained within international clinical practice guidelines for low back pain. Resources also detail guideline recommended advice about the nature of the condition, the diagnosis, prognosis and evidence-based treatments, as well as common misconceptions about back pain and its management.

\section{Control group}

Participants randomised to the control group will continue on the usual care pathway and take part in data collection during the 6 month intervention period. Currently no active management of low back pain patients waiting for an outpatient orthopaedic consultation occurs. Control group patients will be informed that a face-to-face appointment to determine the need for further care will be available in 6 months.

\section{Data collection}

Participants will be followed for 6 months (26 weeks) and be asked to complete three self-reported questionnaires at baseline (pre-randomisation), week 6 and 26 weeks post randomisation to collect primary and secondary outcome data. All participants will be mailed a questionnaire one week prior to the 6 and 26 week time point and then asked to provide responses in one of two ways: via telephone or returned postal questionnaire. The baseline questionnaire will be completed via telephone only. Participants will also be asked to record the primary outcome 'pain intensity' at week 2, 10, 14, 18 and 22. Participants will be asked to provide these data via telephone or reply to text message, whichever their preference. During the 26 week telephone survey participants will be asked to attend a follow up clinic appointment (intervention group) or initial clinical appointment (control group) with a health professional.

\section{Measures \\ Baseline demographic characteristics}

The following demographic items will be collected at baseline: age, Aboriginal and/or Torres Strait Islander status, employment status, country of origin, highest level of education, health insurance status and medical conditions. Length of time waiting for consultation (days) and triage classification will be obtained from hospital records.

\section{Primary outcome}

Pain intensity Pain intensity will be measured using a 0-10 NRS, as the average pain over the last week where zero indicates 'no pain' and ten indicates the 'worst possible pain' [26]. Pain intensity will be collected at baseline, at $2,6,10,14,18,22$ and 26 weeks post randomisation (see Table 1). The NRS is a valid and reliable measure of pain intensity in adults with low back pain [27].

\section{Secondary outcomes}

The secondary outcomes include: low back pain disability, using the Roland Morris Disability Questionnaire (RMDQ) [28]; self-reported weight $(\mathrm{kg})$; objective weight $(\mathrm{kg})$ measured to the nearest $0.1 \mathrm{~kg}$ by a trained assessor using 
Table 1 Trial Measures

\begin{tabular}{|c|c|c|c|}
\hline Outcome & Domain & Measures & Time point (weeks) \\
\hline Primary & Pain intensity & $\begin{array}{l}\text { Pain intensity over the previous week as measured by the } 0-10 \\
\text { Numerical Rating Scale (NRS) [26] }\end{array}$ & $0,2,6,10,14,18,22,26$ \\
\hline \multirow[t]{21}{*}{ Secondary } & Disability & Roland Morris Disability Questionnaire (RMDQ) [28] & $0,6,26$ \\
\hline & Self-reported weight & Self-reported weight (kg) & $0,6,26$ \\
\hline & Objective weight & Measured to the nearest $0.1 \mathrm{~kg}$ [29] & $0^{\mathrm{a}}, 26$ \\
\hline & BMl & Calculated as weight/height squared $\left(\mathrm{kg} / \mathrm{m}^{2}\right)$ & $0^{\mathrm{a}}, 26$ \\
\hline & Waist circumference & Measured to the nearest $0.1 \mathrm{~cm}[29]$ & 26 \\
\hline & Quality of life & Short Form 12 version 2 (SF12.v2) [30] & $0,6,26$ \\
\hline & Perceived change in condition & Global Perceived Effect Scale [43] & 6,26 \\
\hline & Psychological distress & Depression, Anxiety and Stress Scale-21 (DASS-21) [31] & 0,26 \\
\hline & Sleep quality & Item 6 of the Pittsburgh sleep quality index [32] & $0,6,26$ \\
\hline & \multirow[t]{4}{*}{ Health behaviours } & Physical Activity measured using the Active Australia Survey [33] & $0,6,26$ \\
\hline & & Dietary intake measured using a short food frequency questionnaire [34] & $0,6,26$ \\
\hline & & $\begin{array}{l}\text { Alcohol Consumption measured using the alcohol use disorders } \\
\text { identification test (AUDIT) [35] }\end{array}$ & $0,6,26$ \\
\hline & & Self-reported smoking status [36] & $0,6,26$ \\
\hline & \multirow[t]{3}{*}{ Health care utilisation } & Medication use for low back pain & $0,6,26$ \\
\hline & & Visits for low back pain - type and number of sessions & $0,6,26$ \\
\hline & & Attended orthopaedic consultation, received surgery & 26 \\
\hline & Pain attitudes & Survey of Pain Attitudes (SOPA) [38] & $0,6,26$ \\
\hline & Fear Avoidance & Fear Avoidance Beliefs Questionnaire (FABQ) [39] & 0,26 \\
\hline & \multirow[t]{3}{*}{ Economic } & Quality of life (SF12.v2) & \multirow[t]{3}{*}{$0,6,26$} \\
\hline & & Health care utilisation (including estimated out of pocket cost) & \\
\hline & & $\begin{array}{l}\text { Absenteeism (days off normal work due to lower back pain in the } \\
\text { past } 6 \text { weeks) }\end{array}$ & \\
\hline
\end{tabular}

GHS: Get Healthy Information and Coaching Service; ${ }^{a}$ Intervention group only

International Society for the Advancement of Kinanthropometry (ISAK) procedures [29]; BMI calculated as weight /height squared $\left(\mathrm{kg} / \mathrm{m}^{2}\right)$; waist circumference measured at 26 weeks post randomisation taken at the level of the narrowest point between the inferior rib border and the iliac crest by trained assessors using a flexible tape measure to the nearest $0.1 \mathrm{~cm}$ [29]; quality of life assessed using the 12item Short Form Health Survey version 2 (SF-12.v2) [30]; global perceived change in condition measured using the Global Perceived Effect Scale ( -5 to 5 scale) [29]; psychological distress using the Depression, Anxiety and Stress Scale-21 (DASS-21) [31]; sleep quality measured using item 6 of the Pittsburgh Sleep Quality Index [32]; health behaviours including physical activity reported as the frequency and total minutes of spent participating in physical activity measured by the Active Australia Survey [33], dietary intake measured by a short food frequency questionnaire (FFQ) [34], alcohol consumption measured using the Alcohol Use Disorders Identification Test (AUDIT) [35] and self-reported current smoking status [36]; health care utilisation including medication use, type of health services utilised for low back pain and the number of sessions [37]; and attitudes and beliefs measured by the Survey of Pain Attitudes (SOPA) [38] and the Fear Avoidance Beliefs Questionnaire (FABQ) [39]. See Table 1 for data collection time points for secondary outcomes.

\section{Intervention and data integrity}

The delivery of the intervention will be assessed using attendance records for the physiotherapy consultation and data regarding delivery of the GHS intervention including, commencement and number, length, timing of coaching calls and achievement of identified goals which will be provided by the GHS. Patient reported receipt of care (as well as additional care) will be collected at all secondary collection time points. Participants will be monitored for adverse events throughout the intervention period. All adverse events will be recorded and serious adverse events will be assessed and managed on a case-by-case basis according to Good Clinical Practice (GCP) guidelines [40]. Trial data integrity will be monitored by regularly scrutinising data files for omissions and errors. Manually entered data (i.e. data not recorded directly by participant) will be double entered 
and the source of any inconsistencies will be explored and resolved in consultation with the lead investigator (CW). Data will be stored on password protected files, with access given to approved researchers only.

\section{Sample size}

Sample size was calculated using Stata sample size calculator. Using a standard deviation of 2.3, a two-sided alpha of 0.025 (to account for multiple outcomes of interest - pain and weight) [41] and allowing for $15 \%$ loss to follow up, a sample of 80 participants per group will provide $90 \%$ power to detect a clinically meaningful difference of 1.5 in pain intensity (pain numerical rating scale) between intervention and control groups at 26 weeks post randomisation. This sample also provides power $80 \%$ to detect a $6 \%$ reduction in weight in the underlying sampling population and based on evidence from other musculoskeletal conditions is hypothesized to lead to a clinically meaningful reduction in pain [42].

\section{Statistical analysis}

\section{Primary outcomes analysis}

Between group differences in pain intensity will be assessed using linear mixed models, with random intercepts for individuals to account for correlation of repeated measures. We will obtain estimates of the effect of the intervention and $95 \%$ confidence intervals by constructing linear contrasts to compare the adjusted mean change in outcome from baseline to each time point between the treatment and control groups. Dummy coded variables representing group allocation will be used to ensure blinding of the analyses. Missing data will be assessed for randomness if this is more than $10 \%$.

\section{Secondary outcomes analysis}

Linear mixed models will be used to assess treatment effects on secondary outcomes as per the primary outcome. We will compare the adjusted mean change (continuous variables) or difference in proportions (dichotomous variables) in outcome from baseline to each time point between the treatment and control groups.

An economic evaluation will also be undertaken. We will develop costing models from the perspective of the health service and broader societal perspective. These models will utilize data regarding patient quality of life (SF12v2), health care and community services use, work absenteeism. We will calculate costs based on published normative data and estimated out of pocket costs reported by participants. We will also investigate the mechanisms underlying the intervention using causal mediation analysis and include the following measures at baseline, 6 weeks and 6 months: pain attitudes (SOPA), fear avoidance beliefs (FABQ) and symptoms of psychological distress (DASS 21), weight loss (kg), health behaviours (physical activity (MVPA), diet, alcohol, smoking, sleep quality).

\section{Discussion}

This is the first RCT designed to evaluate the effectiveness of a lifestyle behavioural intervention for low back pain patients who are overweight or obese. The results will inform care pathways by providing robust evidence about the effectiveness of such management for overweight patients with low back pain.

\section{Abbreviations}

BMI: Body Mass Index; RCT: Randomised controlled trial; ODI: Oswestry Disability Index; SPIRIT: Standard Protocol Items: Recommendations for Intervention Trials; NSW: New South Wales; NRS: Numerical rating scale; GHS: NSW Get Healthy Information and Coaching Service; RMDQ: Roland Morris Disability Questionnaire; ISAK: International Society for the Advancement of Kinanthropometry; SF12.v2: Short Form Healthy Survey version 2; DASS-21: Depression Anxiety Stress Scale-21; FFQ: Food Frequency Questionnaire; AUDIT: Alcohol Use Disorders Identification Test; SOPA: Survey of Pain Attitudes; FABQ: Fear Avoidance Beliefs Questionnaire; GCP: Good Clinical Practice; HMRI: Hunter Medical Research Institute.

\section{Competing interests}

The authors declare that there are no competing interests.

\section{Author's contributions}

AW, CW, KO, JW, LW, SY, LC were responsible for the design of the study. CW and JW procured funding. All authors contributed to developing the intervention and data collection protocols and materials, and reviewing, editing, and approving the final version of the paper. AW drafted the manuscript and all authors have contributed to the manuscript. All authors have read and approved the final manuscript.

\section{Acknowledgements}

This study is funded by Hunter New England Local Health District and the Hunter Medical Research Institute (HMRI). The project also received infrastructure support from the University of Newcastle.

\section{Author details}

'Hunter New England Population Health, Locked Bag 10, Wallsend NSW, 2287, Australia. ${ }^{2}$ Hunter Medical Research Institute, Locked Bag 1, Hunter Region Mc, NSW 2310 Newcastle, Australia. ${ }^{3}$ University of Newcastle, Newcastle 2308, Australia. ${ }^{4}$ Neuroscience Research Australia, PO Box 1170, Randwick NSW 2031, Australia. ${ }^{5}$ Prince of Wales Clinical School, University of New South Wales, Randwick 2031, Australia. ${ }^{6}$ Ambulatory Care Centre, John Hunter Hospital, Hunter New England Local Health District, Locked Bag 664 J, Newcastle NSW 2300, Australia. ${ }^{7}$ The George Institute for Global Health, University of Sydney, PO Box M201, Sydney NSW 2050, Australia.

Received: 30 November 2015 Accepted: 3 February 2016

Published online: 11 February 2016

\section{References}

1. Hoy D, Bain C, Williams G, March L, Brooks P, Blyth F, et al. A systematic review of the global prevalence of low back pain. Arthritis Rheum. 2012;64:2028-37.

2. Balagué F, Mannion AF, Pellisé F, Cedraschi C. Non-specific low back pain. Lancet. 2012:379:482-91.

3. Vos T, Barber RM, Bell B, Bertozzi-Villa A, Biryukov S, Bolliger I, et al. Global, regional, and national incidence, prevalence, and years lived with disability for 301 acute and chronic diseases and injuries in 188 countries, 1990-2013: a systematic analysis for the Global Burden of Disease Study 2013. Lancet. 2015:386:743-800.

4. Arthritis and Osteoporosis Victoria. A Problem worth solving - The rising cost of musculoskeletal conditions in Australia. Elsternwick: Arthritis and Osteoporosis Victoria; 2013. 
5. Dagenais S, Caro J, Haldeman S. A systematic review of low back pain cost of illness studies in the United States and internationally. Spine J. 2008;8:8-20. Off. J. North Am. Spine Soc.

6. Leboeuf-Yde C. Body weight and low back pain. A systematic literature review of 56 journal articles reporting on 65 epidemiologic studies. Spine. 2000;25:226-37.

7. Shiri R, Karppinen J, Leino-Arjas P, Solovieva S, Viikari-Juntura E. The association between obesity and low back pain: a meta-analysis. Am J Epidemiol. 2010;171:135-54.

8. Heuch II, Hagen K, Zwart J-A. Body mass index as a risk factor for developing chronic low back pain: a follow-up in the Nord-Trø ndelag Health Study. Spine. 2013;38:133-9.

9. National Health and Medical Research Council. Clinical Practice Guidelines for the Management of Overweight and Obesity in Adults, Adolescents and Children in Australia. National Health and Medical Research Council; 2013.

10. Wai EK, Rodriguez S, Dagenais S, Hall H. Evidence-informed management of chronic low back pain with physical activity, smoking cessation, and weight loss. Spine J. 2008;8:195-202. Off. J. North Am. Spine Soc.

11. Roffey DM, Budiansky A, Coyle MJ, Wai EK. Obesity and Low Back Pain: Is There a Weight of Evidence to Support a Positive Relationship? Curr Obes Rep. 2013:2:241-50.

12. Linton SJ, van Tulder MW. Preventive interventions for back and neck pain problems: what is the evidence? Spine. 2001;26:778-87.

13. Roffey DM, Ashdown LC, Dornan HD, Creech MJ, Dagenais S, Dent RM, et al. Pilot evaluation of a multidisciplinary, medically supervised, nonsurgical weight loss program on the severity of low back pain in obese adults. Spine J. 2011;11:197-204.

14. Graves N, Barnett AG, Halton KA, Veerman JL, Winkler E, Owen N, et al. Cost-effectiveness of a telephone-delivered intervention for physical activity and diet. PLoS One. 2009:4:e7135.

15. Goode AD, Reeves MM, Eakin EG. Telephone-delivered interventions for physical activity and dietary behavior change: an updated systematic review. Am J Prev Med. 2012;42:81-8.

16. Appel LJ, Clark JM, Yeh H-C, Wang N-Y, Coughlin JW, Daumit G, et al. Comparative effectiveness of weight-loss interventions in clinical practice. N Engl J Med. 2011:365:1959-68.

17. Relton C, Torgerson D, O'Cathain A, Nicholl J. Rethinking pragmatic randomised controlled trials: introducing the 'cohort multiple randomised controlled trial' design. BMJ. 2010;340:963-7.

18. Krismer M, van Tulder M. Strategies for prevention and management of musculoskeletal conditions. Low back pain (non-specific). Best Pract Res Clin Rheumatol. 2007;21:77-91.

19. O'Hara BJ, Bauman AE, Eakin EG, King L, Haas M, Allman-Farinelli M, et al. Evaluation Framework for Translational Research: Case Study of Australia's Get Healthy Information and Coaching Service ${ }^{\circledast}$. Health Promot Pract. 2013;14(3):380-9

20. O'Hara BJ, Phongsavan P, Venugopal K, Eakin EG, Eggins D, Caterson H, et al. Effectiveness of Australia's Get Healthy Information and Coaching Service ${ }^{\oplus}$. translational research with population wide impact. Prev Med. 2012;55:292-8

21. Ryan RM, Deci EL. Self-determination theory and the facilitation of intrinsic motivation, social development, and well-being. Am Psychol. 2000;55:68-78,

22. Silva MN, Markland D, Minderico CS, Vieira PN, Castro MM, Coutinho SR, et al. A randomized controlled trial to evaluate self-determination theory for exercise adherence and weight control: rationale and intervention description. BMC Public Health. 2008:8:234.

23. Brown WJ, Bauman AE, Bull FC, Burton NW. Development of Evidence based Physical Activity Recommendations for Adults (18-64 years). Report prepared for the Australian Government Department of Health; 2012 Aug; Available from: http://www.health.gov.au/internet/main/publishing.nsf/ Content/health-pubhlth-strateg-phys-act-guidelies/\$File/DEB-PAR-Adults-1864years.pdf.

24. Rollnick S, Miller WR. What is motivational interviewing? Behav Cogn Psychother. 1995;23:325-34.

25. Palmer S, Tubbs I, Whybrow A. Health coaching to facilitate the promotion of healthy behaviour and achievement of health-related goals. Int J Health Promot Educ. 2003:41:91-3.

26. Von Korff M, Ormel J, Keefe FJ, Dworkin SF. Grading the severity of chronic pain. Pain. 1992;50:133-49.

27. Hawker GA, Mian S, Kendzerska T, French M. Measures of adult pain: Visual Analog Scale for Pain (VAS Pain), Numeric Rating Scale for Pain (NRS Pain), McGill Pain Questionnaire (MPQ), Short-Form McGill Pain Questionnaire
(SF-MPQ), Chronic Pain Grade Scale (CPGS), Short Form-36 Bodily Pain Scale (SF-36 BPS), and Measure of Intermittent and Constant Osteoarthritis Pain (ICOAP). Arthritis Care Res. 2011;63:240-52.

28. Roland $M$, Morris R. A study of the natural history of back pain: part l: development of a reliable and sensitive measure of disability in low-back pain. Spine. 1983;8:141-4.

29. International Society for the Advancement of Kinanthropometry. International Standards for Antrhopometric Assessment. Underdale: ISAK; 2001.

30. Ware JE, Kosinski M, Turner-bowker DM, Gandek B. User's Manual for the SF12v2 Health Survey (With a Supplement Documenting SF-12 Health Survey). Lincoln: QualityMetric Incorporated; 2002.

31. Lovibond PF, Lovibond SH. The structure of negative emotional states: Comparison of the Depression Anxiety Stress Scales (DASS) with the Beck Depression and Anxiety Inventories. Behav Res Ther. 1995;33:335-43.

32. Buysse DJ, Reynolds CF, Monk TH, Berman SR, Kupfer DJ. The Pittsburgh Sleep Quality Index: a new instrument for psychiatric practice and research. Psychiatry Res. 1989;28:193-213.

33. Australian Institute of Health and Welfare (AlHW). The Active Australia Survey: a guide and manual for implementation, analysis and reporting. Canberra: AlHW; 2003

34. Centre for Epidemiology and Research. NSW Population Health Survey. Sydney: NSW Department of Health; 2014

35. Babor T, Higgins-Biddle J, Saunders J, Monteiro M. AUDIT. The alcohol use disorders identification test: guidelines for use in primary care (2nd Ed). Geneva: World Health Organisation; 1992

36. Shiri R, Karppinen J, Leino-Arjas P, Solovieva S, Viikari-Juntura E. The association between smoking and low back pain: a meta-analysis. Am J Med. 2010;123:87. e7-35.

37. Williams CM, Latimer J, Maher CG, McLachlan AJ, Cooper CW, Hancock MJ, et al. PACE-the first placebo controlled trial of paracetamol for acute low back pain: design of a randomised controlled trial. BMC Musculoskelet Disord. 2010;11:169.

38. Jensen MP, Karoly P, Huger R. The development and preliminary validation of an instrument to assess patients' attitudes toward pain. J Psychosom Res. 1987;31:393-400.

39. Waddell G, Newton M, Henderson I, Somerville D, Main CJ. A FearAvoidance Beliefs Questionnaire (FABQ) and the role of fear-avoidance beliefs in chronic low back pain and disability. Pain. 1993;52:157-68.

40. Department of Health and Ageing Therapeutic Goods Administration. The Australian clinical trial handbook: a simple, practical guide to the conduct of clinical trials to international standards of good clinical practice (GCP) in the Australian context [Internet]. Canberra: Commonwealth of Australia; 2006 [cited 2015 Sep 20]; Available from: http://www.australianclinicaltrials.gov. au/researchers/good-clinical-practice-gcp-australia.

41. Proschan MA, Waclawiw MA. Practical guidelines for multiplicity adjustment in clinical trials. Control Clin Trials. 2000;21:527-39.

42. Christensen $\mathrm{R}$, Bartels EM, Astrup A, Bliddal H. Effect of weight reduction in obese patients diagnosed with knee osteoarthritis: a systematic review and meta-analysis. Ann Rheum Dis. 2007:66:433-9.

43. Kamper SJ, Ostelo RWJG, Knol DL, Maher CG, de Vet HCW, Hancock MJ. Global Perceived Effect scales provided reliable assessments of health transition in people with musculoskeletal disorders, but ratings are strongly influenced by current status. J Clin Epidemiol. 2010;63:760-6.

\section{Submit your next manuscript to BioMed Central and we will help you at every step:}

- We accept pre-submission inquiries

- Our selector tool helps you to find the most relevant journal

- We provide round the clock customer support

- Convenient online submission

- Thorough peer review

- Inclusion in PubMed and all major indexing services

- Maximum visibility for your research

Submit your manuscript at www.biomedcentral.com/submit 